\title{
Integration of accurate 2D inundation modelling, vector land use database and economic damage evaluation
}

\section{J. Ernst}

Research Unit of Hydrology, Applied Hydrodynamics and Hydraulic Constructions (HACH), Department ArGEnCo, University of Liege, Belgium

\section{B. J. Dewals}

Research Unit of Hydrology, Applied Hydrodynamics and Hydraulic Constructions (HACH), Department ArGEnCo, University of Liege, Belgium \& Postdoctoral Researcher of the Fund for Scientific Research, F.R.S.-FNRS, Belgium

\author{
P. Archambeau; S. Detrembleur; S. Erpicum \& M. Pirotton \\ Research Unit HACH, Department ArGEnCo, University of Liege, Belgium
}

ABSTRACT: Within the global framework of the climate change, according to most Regional Climate Models the maximum peak discharges in river will increase in importance and frequency. Therefore the people will have to face worst inundation conditions. In order to protect themselves from the increasing flood risk, the communities have to draw up suitable flood protection measures. The selection and the evaluation among the different possible flood mitigation measures requires developing decision-support system (DSS). This system has to take into consideration parameters such as hydraulic, economic, social or environmental. This paper focuses on the integration between two components of the DSS, namely the evaluation of the economic impacts of floods and the hydrodynamic modelling. The hydraulic simulations are conducted by means of WOLF $2 D$ flow modelling system and provide as an output high resolution flood maps detailing the distribution of water depth and flow velocity in the floodplains. The integration is ensured by the use of very accurate geographic databases and an automated procedure which makes the most of geomatic methods. The paper details the application of the integrated assessment procedure for a case study along the river Ourthe located in the Meuse basin (Belgium). Moreover, possibilities of validation of the economic damage evaluation procedure are investigated by comparing computation results with real damage data recorded by the Belgian Disaster Fund after several major flood events (2003, 2002, 1995, and 1993).

\section{INTRODUCTION}

\subsection{Context}

In Belgium, the national research project "ADAPT Towards an integrated decision tool for adaptation measures", aims at developing a decision-support system (DSS) dedicated to the integrated evaluation of flood protection measures in the context of increased flooding hazard as a result of climate change. This DSS is based on a combination of costbenefit analysis (CBA) and multi-criteria analysis (MCA) and takes into consideration hydraulic, economic, social as well as environmental parameters.

The present paper focuses on the integration between two components of the DSS, namely the hydrodynamic modelling and the evaluation of the economic impacts of floods. The methodology is demonstrated for a study area located along the river Ourthe in the Meuse basin (Belgium). The hydrodynamic simulations for this case study are conducted by means of $2 \mathrm{D}$ flow modelling and provide as an output high resolution flood maps detailing the 2D distribution of water depth and flow velocity in the floodplains.
Besides a brief overview of the hydrodynamic modelling approach, the paper details the integrated assessment procedure and its application for the case study on the river Ourthe. In particular, the validation of the economic damage evaluation procedure is depicted as the comparisons between computed results and real damage data recorded by the Belgian Disaster Fund after recent flood events (2003, 2002, 1995, and 1993) (§ 6).

The originality of the method of integration suggested in this paper is to propose a micro scale analysis on wide simulation domain. But contrary to the majority of this type of analysis, it is not used an object oriented method. The analysis resolution is even more detailed since the resolution of the economic analysis is the same that the hydrodynamic simulations, i.e. a 2 meters Cartesian grid.

In this present paper relative damage functions are exploited. They link the hydraulic parameters and the percentage of damage, are up to now derived from those reported in several literature references (e.g. ICPR Rhine atlas, MEDIS project), while the specific value of the elements-at-risk is gathered from various sources, such as local authorities or Land Registry offices. 


\subsection{Overview of the methodology}

As shown in Figure 1, flood risk is defined by the sum of the product of probability by consequences. The probability that a flood event occurs can be derived from its statistical return period (in years). The discharge corresponding to the statistical return period is the probability that this discharge is at least exceeded during this period, and, by differentiation, the probability that this discharge will be observed can be deduced. The consequences are defined by three parameters: (i) the exposure, expressed by the hydraulic modelling results (e.g. inundation extent, water depth, flow velocity, etc.); (ii) the elements-atrisk, the assets which would be affected by a flood event; (iii) the vulnerability, describe the resilience, the adaption ability and, the susceptibility of the elements-at-risk.

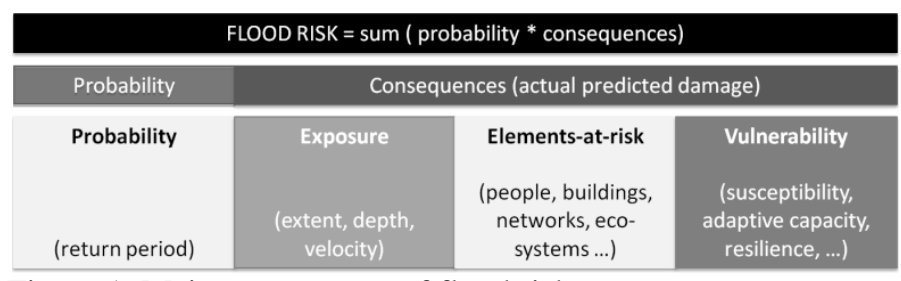

Figure 1. Main components of flood risk.

Within the framework of the Belgian project ADAPT, this paper will develop an original methodology devoted to the consequences assessment, in particular the tangible and direct ones. Its aim is to provide tools for selecting and assessing individual flood protection measures (e.g. protection wall building, passive floodplain activation, etc.).

The special technical features of this method are that it is devoted to very detailed analysis by combining a 2D distributed flow model based on the complete shallow flow equations ( $\S 3$ ), very accurate spatial databases ( $\S 4$ ), such as Digital Elevation Model, and land use data.

\section{LITERATURE REVIEW}

While speaking about flood management, the terms macro, meso, and micro scale level analysis are usually employed (FloodSite 2006):

- macro-scale methods consider land use administrative, locality scale unit;

- meso-scale methods consider also land use unit as analysis resolution but distributed land use i.e. residential area, industrial, etc.;

- micro-scale methods usually apply an object oriented approach, each building is considered individually.

Although these terms refer to a spatial resolution of the analysis, with the recent improvements of the geographic land use databases (Van der Sande et al. 2003), several flood damage assessment methods object oriented analysis are also applied on large ex- tent, regional or event national. As a result, the prefixes (macro, meso, micro) should be used for identifying the general methodology approach of the damage assessment and not for suggesting the spatial resolution of the analysis.

Damage functions are an essential component in the general methodology of an economic damage assessment. These functions establish the link between the modelled hydraulic parameters, i.e. water depth, duration, flow velocities, water contamination, etc. and the damage generated to goods affected by these hydraulic constraints. But a major issue which intervenes in the fitting of such functions is the lack of data of validation. In Germany, within the framework of the project MEDIS, information has been extensively collected campaigns among the population affected by the principal floods which occurred in this country. Although the quantity of data is rather important in this case, the only parameter influences the damage sudden is the water level. The reason is that other flood characteristics are rather not easily measurable. However, certain authors included the water level contamination (Thieken et al. 2008), or else flood duration in their analysis (Penning-Rowsell et al. 2003) by distinguishing two categories: short (less than 12 hours) and long (more than 12 hours) duration of flooding.

After this literature review, the characteristics of the methodology described in this paper will be defined and compared to the existing methodologies in the following. The major particularity of the model is the resolution scale and the hydraulic and economic integration. The model is a micro scale analysis with very high resolution (i.e. at the hydraulic modelling resolution), and as mentioned below, the economic analysis only concerns the direct and tangible damage assessment. Contrary to most of the micro scale flood damage assessment put forward by the scientific community. This damage evaluation is not an object-oriented method (damage calculated for single assets) but the analysis goes further in detail, i.e. 2 meters analysis grid. It means that each computational mesh is subject to the damage assessment process ( $\S 5$ ). This feature leads to very precise of flood protection measures assessment such as protection wall construction or passive floodplain activation.

\section{HYDRAULIC MODEL}

It is shown that interactions between the minor and the floodplain of rivers during flooding are important and cannot be neglected. Simplified 1D flow models which are usually used as hydrodynamic input data for flood loss estimation are unfortunately not able to model these transfers between these two areas of the flow. Moreover by the unceasingly increasing need of damage quantification following 
floods and in particular in the urban or strongly urbanized areas, the assumptions of one dimensional model are not acceptable any more. In this paper, with the aim of modelling the interactions between the minor bed and the major bed and also to reproduce most accurately the flows in complex topographic structures such as urban areas, a hydrodynamic 2D model is used.

The HACH research unit from the University of Liege develops a wide range of numerical tools for simulating free surface flows and transport phenomena. Those computational models are interconnected and integrated within one single modelling system named WOLF. Among those computation units, the two-dimensional flow model WOLF $2 D$ is exploited for the present study and is described hereafter. $W O L F 2 D$ is based on the depth-averaged equations of volume and momentum conservation, i.e. the “shallow-water” equations (Chaudhry 1993):

$\frac{\partial \vec{s}}{\partial t}+\frac{\partial \vec{f}}{\partial x}+\frac{\partial \vec{g}}{\partial y}+\frac{\partial \vec{f}_{\mathrm{d}}}{\partial x}+\frac{\partial \vec{g}_{\mathrm{d}}}{\partial y}=\vec{S}_{0}-\vec{S}_{\mathrm{f}}$

with $\vec{s}=\left[\begin{array}{lll}h & h u & h v\end{array}\right]^{\mathrm{T}}$ the vector of the conservative unknowns. $\vec{S}_{0}$ and $\vec{S}_{\mathrm{f}}$ designate respectively the bottom slope term and the friction term:

$$
\begin{aligned}
& \vec{S}_{0}=-g h\left[\begin{array}{lll}
0 & \partial z_{\mathrm{b}} / \partial x & \partial \mathrm{z}_{\mathrm{b}} / \partial y
\end{array}\right]^{\mathrm{T}} \\
& \vec{S}_{\mathrm{f}}=\left[\begin{array}{llll}
0 & \tau_{\mathrm{b}, x} / \rho & \tau_{\mathrm{b}, y} / \rho
\end{array}\right]^{\mathrm{T}}
\end{aligned}
$$

$\vec{f}$ and $\vec{g}$ represent the advective and pressure fluxes in directions $x$ and $y$, while $\vec{f}_{\mathrm{d}}$ and $\vec{g}_{\mathrm{d}}$ are the diffusive fluxes.

Written out in full, these fluxes become:

$$
\begin{aligned}
& \vec{f}=\left(\begin{array}{c}
h u \\
h u^{2}+\frac{1}{2} g h^{2} \\
h u v
\end{array}\right), \vec{f}=\left(\begin{array}{c}
h u \\
h u^{2}+\frac{1}{2} g h^{2} \\
h u v
\end{array}\right), \\
& \vec{g}=\left(\begin{array}{c}
h v \\
h u v \\
h v^{2}+\frac{1}{2} g h^{2}
\end{array}\right), \vec{g}_{\mathrm{d}}=-\frac{h}{\rho}\left(\begin{array}{c}
0 \\
\tau_{x y} \\
\sigma_{y}
\end{array}\right) .
\end{aligned}
$$

The following notations have been used: $t$ represents the time, $x$ and $y$ the space coordinates, $h$ the water depth, $u$ and $v$ the depth-averaged velocity components, $z_{\mathrm{b}}$ the bottom elevation, $g$ the gravity acceleration, $\rho$ the density of water, $\tau_{\mathrm{b} x}$ and $\tau_{\mathrm{b} y}$ the bottom shear stresses, $\sigma_{x}$ and $\sigma_{y}$ the turbulent normal stresses, and $\tau_{x y}$ the turbulent shear stress. The bottom friction is conventionally modelled thanks to an empirical law, such as the Manning formula. The models enable the definition of a spatially distributed roughness coefficient. The internal friction may be reproduced by different turbulence closures included in the modelling system, such as simple alge- braic ones but also a complete depth-averaged $k-\varepsilon$ model.

The numerical model deals with multiblock Cartesian grids. This feature increases the size of possible simulation domains and enables local mesh refinements, while preserving the lower computation cost required by Cartesian grids compared to unstructured ones. A grid adaptation technique restricts the simulation domain to the wet cells. Besides, wetting and drying of cells is handled free of volume conservation error (Erpicum 2006).

The space discretization is performed by means of a finite volume scheme. Variable reconstruction at cells interfaces is performed linearly, in combination with slope limiting, leading to a second-order spatial accuracy. The advective fluxes are computed by a Flux Vector Splitting (FVS) technique developed by the authors. A Von Neumann stability analysis has demonstrated the stability of this FVS (Dewals 2006). The diffusive fluxes are legitimately evaluated by means of a centred scheme. Since the model is applied to compute steady-state solutions, the time integration is performed by means of a 3step first order accurate Runge-Kutta algorithm, providing adequate dissipation in time. The time step is constrained by the Courant-Friedrichs-Levy (CFL) condition based on gravity waves. A semiimplicit treatment of the bottom friction term is used, without requiring additional computational costs.

The model has been extensively validated and has shown its efficiency for numerous practical applications, (Dewals 2006; Dewals et al. 2006; Dewals et al. 2008c; Erpicum 2006) notably flood modelling (Dewals et al. 2008a).

\section{INPUT DATA}

\subsection{Integration}

In order to combine suitably WOLF $2 D$ hydrodynamic results and economic assessment by a micro scale method, very precise geographic data sources are required. Moreover, in the aim of reducing the time dedicated to field survey often needed for those analyse, very accurate land use databases and economic sources have been exploited. This kind of datasets will allow the overall procedure to be refined and automated.

In the following paragraphs, the hydrologic input of the hydraulic modelling and the significance of the geometric and semantic characteristics from each geographic databases used will be explained and described in details. 


\subsection{Hydrologic input}

Hydrological data used within the framework of this study has been collected from the discharge observations performed at a gauging station on the river Ourthe for a 30 years period. The statistical distribution used for the relation between discharge $(x)$ and frequency is a 2 parameters (i.e. $\alpha, \lambda$ ) Gamma distribution fitted by the maximum likelihood method. In this case the two parameters values are $\alpha=0.01464$, $\lambda=5.80874$. The analytical probability density function expression is shown bellow (Equation 4).

In hydrology, the cumulative distribution function $F(x)$ is more commonly used than $f(x)$. Basically the cumulative function may be defined by the integral from zero to the discharge $x$ of the cumulative function, $f(x)$. This cumulative probability means that the probability that this event occurred (in this case this discharge is observed) is at least reached. This function may also be expressed by the return period, $T$, of a given event, the Equation 5 leads to this value.

$$
\begin{aligned}
& f(x)=\frac{\alpha^{\lambda}}{\Gamma(\lambda)} x^{\lambda-1} e^{-\lambda x} \\
& \text { with }: \Gamma(\gamma)=\int_{0}^{\infty} u^{\gamma-1} e^{-u} d u \\
& F(x)=\frac{1}{T}-1
\end{aligned}
$$

The figure below (Figure 1) illustrates the cumulative frequency Gamma function fitted by the maximum likelihood method as well as the historical data which were used for the fitting (grey dots). The dotted grey curves represent the associated 0.95 confidence intervals, and the black dots on the curve represent the whole of the discharges which have been simulated with the hydraulic model. These discharge values are distributed on the curve in a rather regular way and covers a range from a value close to the main discharge causing no inundation until an extreme value corresponding to just less than a 1500 years return period.

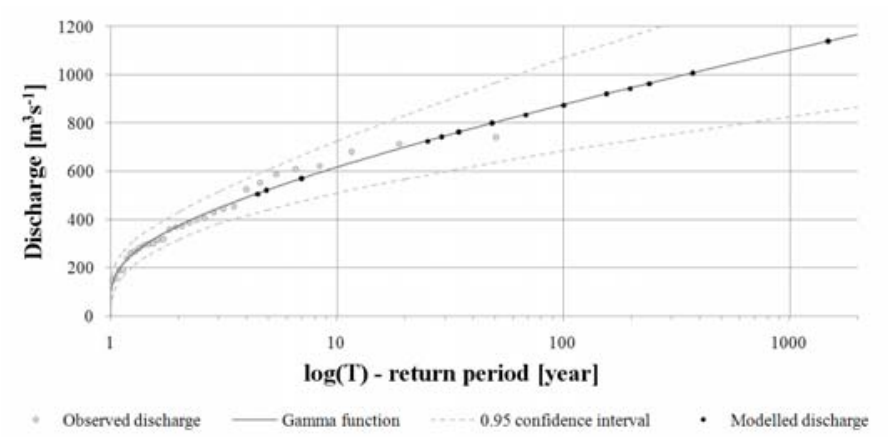

Figure 2. Hydrologic input, discharge related to return period.

\subsection{Digital topographic model}

The remote sensing tool, LiDAR - Light Detection And Ranging is very widespread in the domain of the numerical flow modelling for digital elevation model generation and in particular for bidimensional hydraulic modelling (essentially due to the geometric characteristics of the created digital model). The LiDAR base principle is to measure time delay between the transmission of a laser pulse from an aerial vector (e.g. airplane, helicopter, etc.) and the detection of the reflected signal. Knowing the vector tridimensional position by combining differential GNSS - Global Navigation Satellite System and INS - Inertial Navigation System measurements, the laser pulse orientation as well as the time delay, the position of the object can be computed. The resulting absolute accuracy of the final surface model generated is estimated out about 15 centimetres in elevation for each pulse measurement.

The major issue of this very useful remote sensing technique is that the digital model generated is a surface one and not a terrestrial one. So a pre processing step is required for removing the over grounded object non-relevant to the flow. The best example is indeed the residual vegetation non permeable to laser pulses which is present on the model, such as the conifers.

\subsection{Land use databases}

In Belgium, there are several geographic land use data producers, the most important of them are: first the Belgian National Geographic Institute - IGN and the Walloon Ministry of Facilities and Transport MET. Both provide very accurate land use vector database, respectively named Top10v-GIS and the PICC. This first one contains 18 layers related to information categories (e.g. land use, structure, hydrography, etc.), and on the other hand the PICC data set which is based on stereoscopic aerial imagery restitution and a post processing enrichment with ancient database such as house number, street name, etc.

Both PICC and Top10v-GIS data are very detailed and accurate complementary spatial database at a very high scale (1:10000). The challenge is to extract the strong points from each database and to combine them in a single data set to identify with more accuracy the assets (geometric quality) and their land use type (semantic quality).

\subsection{Land registry}

The Land Registry lists all private property and the value of the goods, the information is also recorded in a geographic database, i.e. each asset listed in this database is located. But because of this register is based sometimes on very old data, its precision is quite lower than the others databases. So the local- 
ization information hold in the Land Registry is not exploited to refine the land use information, i.e. PICC and Top10v-GIS (§ 4.4).

The whole of the geographical data sets previously defined and explained in the preceding paragraphs was devoted to space and building use identification of the objects subjected to the risk of flooding. In contrast, the Land Registry data is used with the single aim of determination of the economic value of the goods. And although these data are also geo referred, the spatial information contained in this database is not exploited to locate the objects, but to be able to assign the value of the goods which are already identified by using the other land use databases. In other terms, the link between this economic data and the land - building use are carried out by a joint on the spatial attribute from several databases.

\section{METHODOLOGY}

The global methodology of economic flood damage assessment proposed in this paper is rather largely widespread. On the other hand, the innovation of the method suggested rests on the fact that each mesh which composes the hydrodynamic simulation domain undergoes the process of evaluation of the damage (Ernst et al. 2008). There is thus no phase of aggregation of the hydraulic two-dimensional modelling result maps which was carried out on a grid with 2 meters of resolution. The distribution of the hydraulic parameters is completely preserved during the application of the economic procedure.

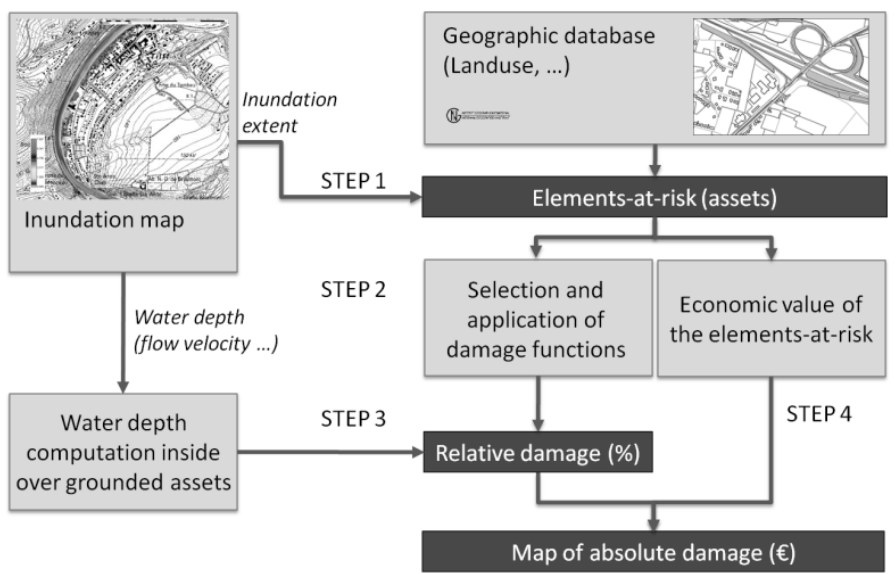

Figure 3. Overview of the four-step process methodology.

The scheme above illustrates the automated fourstep methodology followed for the absolute damage mapping due to a given flood event. In the next paragraphs, each step will be developed and explained in more detail ( $\S$ 5.1, 5.2, 5.3 and 5.4).

\subsection{Step1}

The first step of the damage assessment methodology is to identify the element-at-risk (i.e. the element, the goods which are affected by the flood event). Fundamentally, it can be made by combining the flood extent mapping and the land use geographic databases. But because the hydraulic modelling is run on a Digital Surface Model (which include over grounded structures relevant to the water flow such as building) and not on a Digital Terrestrial Model the identification terms out to be more complex.

A land use type is assigned to each mesh included in the simulation domain. Once the mesh is assigned agriculture or road network land use type, the identification of the elements-at-risk is trivial. But if the mesh is identified as building, the water level computed on it is zero because the hydraulic modelling is run on a DSM (excepting when the flow overtop the building, dam breaking or dike failure scenarios modelling for example). In this case, the assumption made is that the meshes corresponding to a building are considered as affected by the flood event if at least one mesh in the neighbouring is wet.

\subsection{Step 2}

There are two main ways for evaluating the economic damage (FloodSite 2006): either the damage functions provide directly an absolute value of damage in monetary terms, or they provide a relative damage in percent. Although the later approach requires the estimation of the value of the elements-atrisk by surface unit (so called: specific value), it is generally preferred because it may be easily transposed from one studied area to another, combined with relevant local evaluations of the specific values. As illustrated in Figure 4, a large number of relative damage functions are presented in literature (Dushmanta et al. 2003) (International Commission for the Protection of the Rhine 2001). As a result of their scattering results, much care is required in the selection and application of any of them.

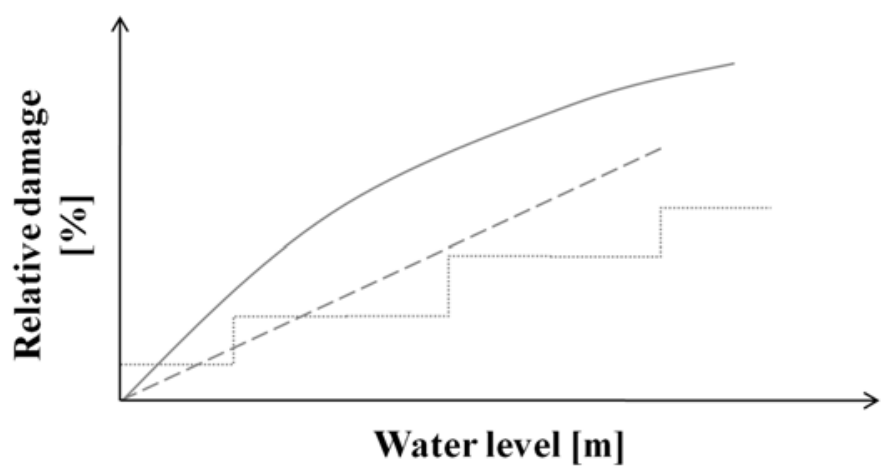

Figure 4. Schematic examples of damage functions.

Recent developments made within the framework of the German MEDIS project have led to reliable 
damage functions setting, called FLEMO - Flood Loss Estimation MOdel. These functions have been fit from dataset gathered by computer-aided phone consultations to household and companies after the major flood events in 2002 in Germany. The FLEMO damage model may be applied on both micro and meso scale economic analysis (Thieken et al. 2008). Some weighting factors may also be used to take into account the local community precaution and the level of water contamination (called FLEMO+).

\subsection{Step 3}

After evaluating the economic parameters (relative damage functions and specific values of the assets) relating to each land and building use, the next step of the process is to compute the relative damage for each asset affected by flooding. As explained in previous paragraphs ( $\S 3$ ), reliable hydraulic 2D modelling has to be run on a Digital Surface Model which includes the structure relevant to the flow to take into account the local effects around the buildings and macro roughness elements. Consequently, the water level inside the over grounded assets such as buildings is not a direct result from the flow modelling, but it has to be computed by a post processing task. Two methods are implemented in the automated process to evaluate the inundation depth of a building:

- first, the average of the wet neighbouring meshes of the assets;

- secondly, the free surface and the ground elevation are interpolated inside the assets by fitting a plan with a least square method, thus a water depth is computed for the whole meshes which compose the assets.

\subsection{Step 4}

Since relative damage functions are preferred to absolute ones, the results of the combination of inundation maps and damage function leads to a relative damage evaluation ( $\S 5.3$ ). Therefore, an additional economic data is needed: the estimated value of the assets affected by the flood. As the analysis is made on each computational mesh, this value is generalized at the specific value (i.e. the value by surface unit $\left.\left[€ / \mathrm{m}^{2}\right]\right)$. Finally, the absolute damage map is computed as the product of the relative damage [\%] by the specific value $\left[€ / \mathrm{m}^{2}\right]$ defined, and by the computational mesh surface $\left[\mathrm{m}^{2}\right]$.

The overall procedure is described at the Figure 5 , the three pictures respectively the first, third and fourth step of the procedure.

1. Identify the elements-at risk (black meshes) by combining the inundation extent maps (grey scaled water depth map in the Figure 5a) and the geographic land use databases;

2. Water depth computation in particular inside the over grounded structures (dark grey meshes), in this case meaning the wet meshes in the vicinity of the object, black meshes) (Figure 5b);

3. Economic losses evaluation by applying damage functions, the economic loss is assessed for each hydraulic computational mesh (grey scale) (Figure 5c).

\section{CASE STUDY AND MODEL VALIDATION}

\subsection{Introduction}

In order to validate the whole of the economic damage assessment methodology, the comparison with former observed events is the most usually used method. Within the framework of this study, the validation data available were collected during the major flood events these last decades in Belgium. Particularly, the zone studied is localized in the Meuse basin on one of the principal tributaries: the river Ourthe. The data available in order to validate this model are detailed below includes both hydrodynamic and economic data.
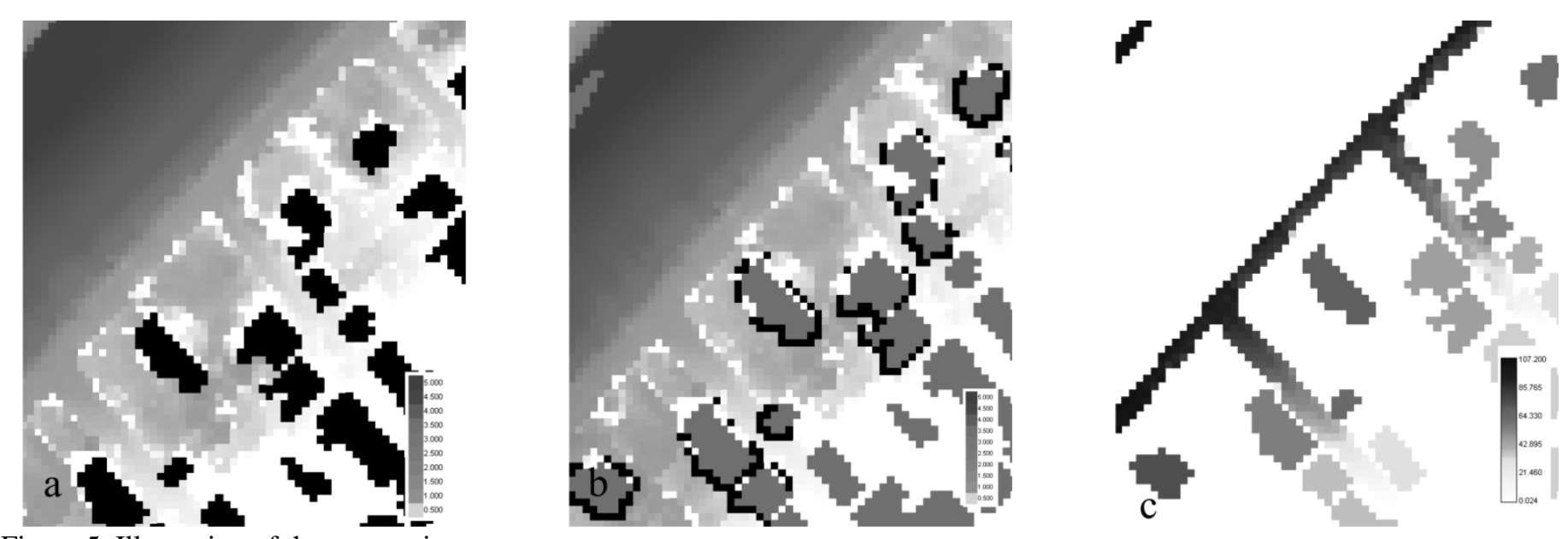

Figure 5. Illustration of the economic assessment process. 


\subsection{Overview of the case study}

With the aim of validating the combined hydraulic and economic damage assessment induced by flooding, the methodology is tested for a case study area, which covers three reaches of the river Ourthe (Figure 6), located respectively $18.5 \mathrm{~km}$ (reach $\mathrm{n}^{\circ} 1$ ), $12.5 \mathrm{~km}$ (reach $\mathrm{n}^{\circ} 2$ ) and $10 \mathrm{~km}$ (reach $\mathrm{n}^{\circ} 3$ ) upstream of the mouth of river Ourthe into river Meuse (Belgium). The total length of the simulated reaches is about $16 \mathrm{~km}$ with a computational Cartesian grid of 2 by 2 meters. Four major past flood events have been modelled: (i) in 1993 a flooding occurred with a maximum peak discharge value: $742 \mathrm{~m}^{3} \mathrm{~s}^{-1}$; (ii) in 1995, $520 \mathrm{~m}^{3} \mathrm{~s}^{-1}$; (iii) in 2002, $570 \mathrm{~m}^{3} \mathrm{~s}^{-1}$; (iv) in 2003, $508 \mathrm{~m}^{3} \mathrm{~s}^{-1}$. Validating data are available concerning the hydrodynamics and the economic part of the method, and are described in detail in the following.

The damage modelling procedure is first applied to past major inundation events, as detailed in § 6.4. Next, prior to eventually assessing the benefits of future flood protection measures to be designed to face climate change, an intermediary step consists in determining how inundation hazard and its economic impact are likely to be modified by climate change. Therefore, hydrodynamic modelling has been carried out to identify how climate change might affect the inundation characteristics (§ 7).

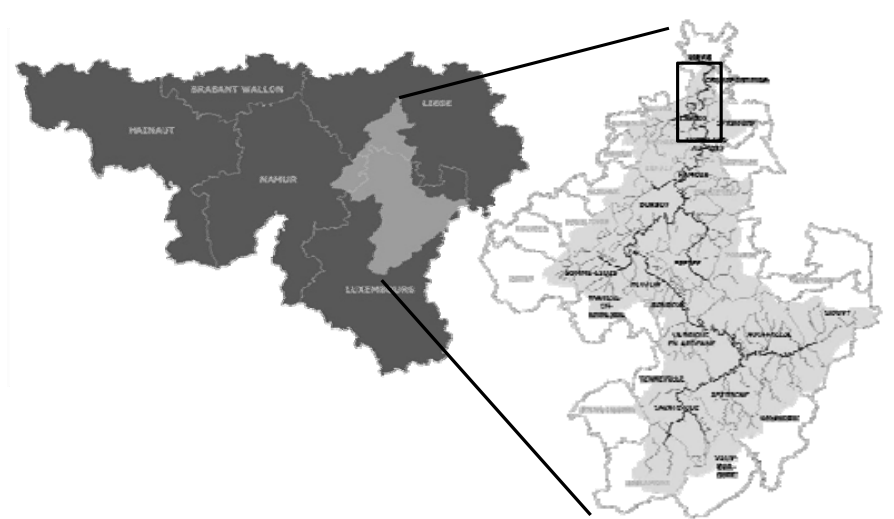

Figure 6. Location of the case study area in the catchment of the river Ourthe.

\subsection{Reference data}

\subsubsection{Field survey}

Although the economic damage assessment methodology related to the flood described in this paper requires no, or very limited, field survey, to carry out a validation of the procedure these terrain surveys often prove to be very useful.

They are needed, on the one hand, for the validation of the topographic model used, since certain topographic characteristics playing a major part in the flow such as protection walls or weirs are present partially or even missing on the initial digital model.
In the absence of other data concerning these characteristics, the recourse to a field survey is quite often essential. On the other hand, this type of study can in addition, be used in order to collect, from the people affected by the flood information about the water level reached in their dwelling and the corresponding damage.

Another data from field survey used is aerial imageries. There were carried out during the major peak discharge in the river. These data give important information concerning the extent of the inundations with the purpose of validating the hydraulic model.

\subsubsection{Disaster Fund}

In Belgium, the Disaster Fund is an organisation which gives compensation to the local community affected by a natural disaster including flooding. The major information recorded in this validating data set concern the validation of the elements-at-risk identification, the first step of the procedure described above ( $§ 5.1$ ) and on the other hand, the absolute damage evaluation. Indeed for each major flood event the sum given to the local communities and the number of housed affected is registered. But for private protection reasons, the sum of the damage suffered by the local community is known street by street only. As the integrated damage assessment presented here is made at a house scale (even at the hydraulic computational mesh size, 2 meters in this case) this information has to be employed very carefully. Further investigation will make possible to extract from this data set aggregated street by street information related to a house individually. This will be possible by combining the two principal sources of information contained in the data of the Disaster Fund data set (i.e. the number of houses and total damage for each street) with the land use information previously described in this paper and finally, the hydraulic results.

\subsection{Validation}

\subsubsection{Hydrodynamic validation}

Even if the use of the different geographic databases reduce significantly the time devoted to field surveys, this task is essential for reliable analysis. The LiDAR is a very precise and reliable remote sensing tool and suitable for digital surface model generation but some topographic particularities are not detected by the laser pulse (e.g. protection walls).

The figure bellow shows a picture of the protection wall (Figure 7a) built downstream of the town Tilff in the aim of protect residential district. The graph next to (Figure $7 \mathrm{~b}$ ) shows the number of houses treated by the flood with or without this protection wall (or if it has not been added to the topographic model). The influence of this topographic re- 
construction is appreciable, principally in the two first class of water depth (§ 7.2).
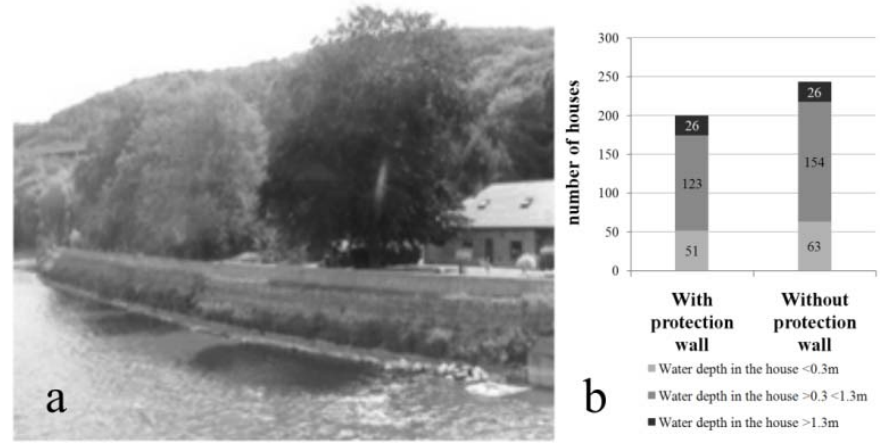

Figure 7. Validation of the topographic model: protection wall influence evaluation.

After this first part of the hydrodynamic validation, other calibrations of the topographic and the hydraulic models may be based on gauging station measurements and also on aerial imagery. Actually, during major flooding, some aerial photographs are taken, on which the extent of the inundation can be easily identified. The comparison of observed flood extents (aerial imageries) and the simulated ones bring some relevant information in order to validate the numeric model. The hydrodynamic model has been already validated for more than 10 years on internationally recognized benchmarks but also on a very large number of practical cases studies. The final precision of the hydrodynamic model is estimated at about 10 centimetres in terms of altitude of free face flow (Erpicum et al. 2008).

\subsubsection{Economic analysis validation}

To validate the damage, the data from the Disaster Fund described in details in the previous chapter will be used ( $\S 6.3 .2)$. The first results of validation are expressed in the graph bellow (Figure 8) and explained in the next paragraph (Dewals et al. 2008b).

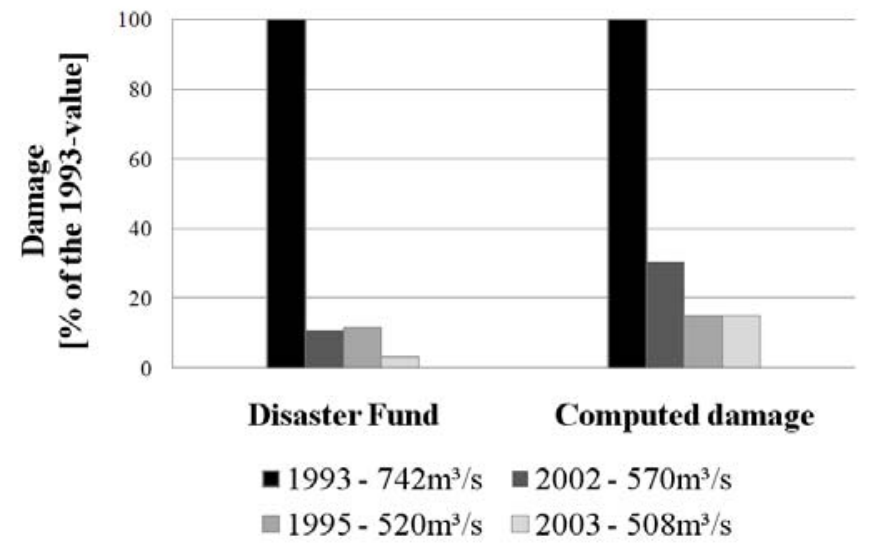

Figure 8. Absolute damage assessment.

The figure above displays the value of goods compensated by the Disaster Fund at the time from the four major floods on the river Ourthe and the damage estimated by the methodology of the dam- age assessment presented in this paper. The correlation between the two graphs is satisfactory knowing that the damage assessment does not take into account the adaptative capacity of people facing a flooding phenomenon. As a result the damage evaluation made for smaller flood events leads to over estimating the total damage considering that the water level is lower, the goods may indeed be easily protected. Contrary to flooding induced by a larger peak discharge for which the traditional protection measures are not applicable any more.

Moreover, it is necessary to keep in mind that all the houses damaged by a flood are not directly compensated by the Disaster Fund. This fact implies that all the houses touched at the time of a rising are not directly compensated. The amount compensated by the Disaster Fund thus clearly underestimated the total sum of damage during the flood.

\section{CLIMATE CHANGE}

\subsection{Main assumptions}

Results of Global Circulation Models (GCM) and Regional Climate Models (RCM) provide estimates of the potential increase in precipitation (mainly during winter and early spring) and potential changes in evapotranspiration as a result of climate change (Boukhris et al. 2007). Those predicted changes are affected by a significant level of uncertainty due to the climate models themselves and, to an even greater extent, to the discrepancies in the scenarios used for running those climate models (Intergovernmental Panel on Climate Change 2007).

At the present stage of the research project, simple assumptions have been considered regarding the expected changes in the peak discharges of the river Ourthe as a result of climate change (CC). Indeed, according to a comprehensive literature review (De Groof et al. 2006; Erpicum et al. 2007), an increase by $10 \%$ of flood discharge may be regarded as reasonable. Moreover, in order to evaluate the sensitivity of the results with respect to this perturbation factor on the discharge, increases by $5 \%$ and by $15 \%$ have also been considered. Finally, a more "extreme" case has been simulated as well $(+30 \%)$. All those assumptions will eventually be confirmed and refined by comparison with the output of a hydrological model (De Groof et al. 2006). The study is performed for two different return periods, namely 25 and 100 years, for which the best flood protection strategies will be complementary, due to the significant differences in inundation extent and in the probability of occurrence. 
Table 1: Discharge and average change in water depth compared to the base scenario, for two return periods and four different climate change (CC) scenarios.

\begin{tabular}{|c|c|c|c|c|c|c|}
\hline & & $\begin{array}{c}\text { Base } \\
\text { scenario }\end{array}$ & $\begin{array}{c}\text { CC scenario } \\
n^{\circ} 1(++5 \%)\end{array}$ & $\begin{array}{l}\text { CC scenario } \\
n^{\circ} 2(+10 \%)\end{array}$ & $\begin{array}{l}\text { CC scenario } \\
n^{\circ} 3(+15 \%)\end{array}$ & $\begin{array}{l}\text { CC scenario } \\
\mathrm{n}^{\circ} 4(+30 \%)\end{array}$ \\
\hline \multirow{3}{*}{$\begin{array}{l}25- \\
\text { year } \\
\text { flood }\end{array}$} & Discharge & $726 \mathrm{~m}^{3} / \mathrm{s}$ & $762 \mathrm{~m}^{3} / \mathrm{s}$ & $799 \mathrm{~m}^{3} / \mathrm{s}$ & $835 \mathrm{~m} / \mathrm{s}$ & $944 \mathrm{~m}^{3} / \mathrm{s}$ \\
\hline & Reach $n^{\circ} 1$ & - & $+10 \mathrm{~cm}$ & $+25 \mathrm{~cm}$ & $+40 \mathrm{~cm}$ & $+75 \mathrm{~cm}$ \\
\hline & Reach $n^{\circ} 2$ & - & $+10 \mathrm{~cm}$ & $+25 \mathrm{~cm}$ & $+40 \mathrm{~cm}$ & $+70 \mathrm{~cm}$ \\
\hline \multirow{3}{*}{$\begin{array}{l}100- \\
\text { year } \\
\text { flood }\end{array}$} & Discharge & $876 \mathrm{~m}^{3} / \mathrm{s}$ & $920 \mathrm{~m}^{3} / \mathrm{s}$ & $964 \mathrm{~m}^{3} / \mathrm{s}$ & $1007 \mathrm{~m} / \mathrm{s}$ & $1139 \mathrm{~m}^{3} / \mathrm{s}$ \\
\hline & Reach $n^{\circ} 1$ & - & $+15 \mathrm{~cm}$ & $+30 \mathrm{~cm}$ & $+45 \mathrm{~cm}$ & $+75 \mathrm{~cm}$ \\
\hline & Reach $n^{\circ} 2$ & . & $+20 \mathrm{~cm}$ & $+40 \mathrm{~cm}$ & $+60 \mathrm{~cm}$ & $+85 \mathrm{~cm}$ \\
\hline
\end{tabular}

\subsection{Results}

Ten discharges corresponding to the reference scenarios (i.e. 25years and 100 years return period) as well as the four probable scenarios of climate change (i.e. an increase of $5,10,15$, and $30 \%$ from the reference cases) were modelled with the hydraulic model. The methodology of evaluation of the damage has been then applied to each hydrodynamic result. Nevertheless, to free it from many uncertainties which appear starting from the economic estimate (step 2) the study is restricted to the identification and the counting of the elements subjected to the risk. The simple assets affected by flooding counting analysis is refined by including three classes of water depth (i.e. under 0.3 metre, between 0.3 and 1.3 meter, over 1.3 meter of water depth). This classification is a standard and widely used for flood mapping in Belgium.

The results of the modelling are summarized at the next two figures (Figure 9, Figure 10). The number of houses affected by the flood related to the different scenarios of climate change is displayed at the Figure 9. For each scenarios (§ 7.1) the figure shows the number of houses in each three classes of water depth.

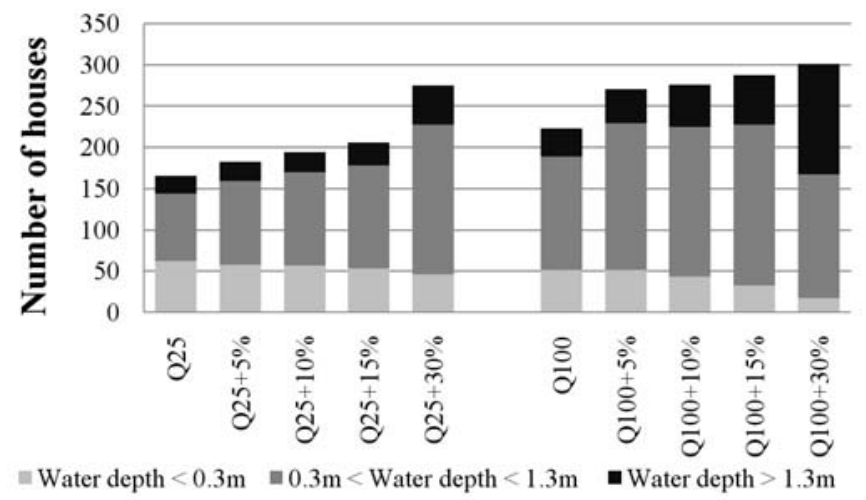

Figure 9. Flood damage evolution in the context of climate change.

The Figure 10 shows the same results but the house count is here related to the return period (in years) of each scenarios. The statistical return period computation is based on the Gamma function fitted without taking into account the climate change ( $\S$ 4.2).

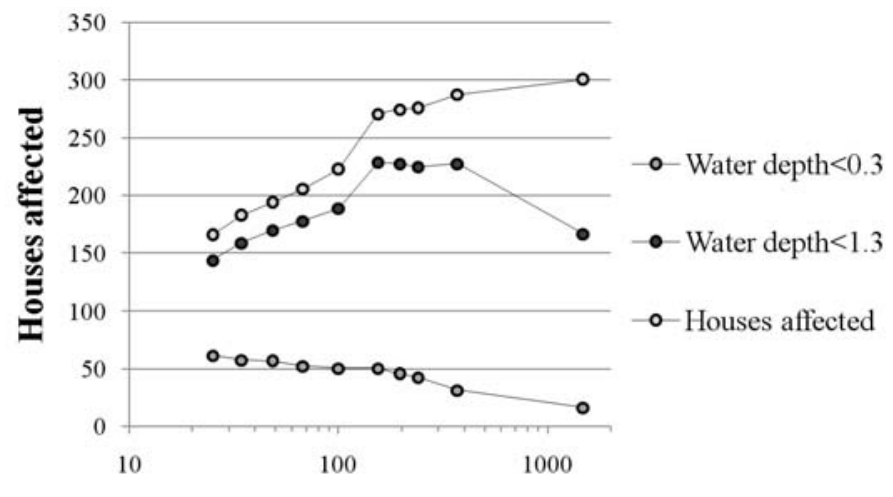

$\log (\mathrm{T})$ - return period [year]

Figure 10. Evolution of the number of houses affected related to the return period (in year).

The graphs analysis reveals that the general tendency is an increase in the number of houses involved in the inundation according to the discharge increase. Nevertheless, in the neighbourhoods of $900 \mathrm{~m}^{3} \mathrm{~s}^{-1}$ or the corresponding 150 years return period an abrupt increase in the total number of houses implicated is noticed. After this rising, the number of house increases but less quickly than before the rising. This fact translates the increasing of the inundation extent.

Without the use of the classes of water depth, the analysis would be limited to these conclusions, but by using of this classification the data extracted from these results are definitely more interesting. Before the rising, the increase of the total number of houses is nearly constant and regular: the extent increases in a steady way with the discharge value. As regard to the classes of water depth, the number of houses in the extreme class remains constant, the increase is observed mainly in the intermediate class (from 0.3 to 1.3 meter of water). On the other hand after the rising the flooded extent does not increase as quickly any more, but the water level increases inside the houses already affected by the floods. More precisely, the number of houses in the higher class (more than 1.3 meter) increases very strongly, and decreases in the two others classes, but especially in the first one.

The whole of these considerations are valid only for the zone of study. However, it would be interesting to identify on other case studies if the same trend is followed upon the application of this methodology.

\section{CONCLUSIONS}

The present paper describes the combination of twodimensional hydrodynamic modelling with a procedure for micro scale economic damage evaluation on large extents in the context of climate change. The high resolution topographic data used for the flow simulations lead to very detailed flood maps, which 
serve as an input for identifying the elements-at-risk and computing the cost of damage, their accuracy ensure to limit the time devoted to field survey. This evaluation is notably based on the following components: detailed vector geographic databases, damage functions and specific values of the elements-at-risk. The overall automatic procedure is first applied to the estimation of the cost of damage caused by a series of real flood events along the river Ourthe (Meuse Basin, Belgium), next it is used to predict the impact of climate change on the expected damage and finally it is exploited for assessing the benefits of adaptation measures. The overall procedure will be further validated and refined, and will subsequently be embedded within a decision-support system dedicated to the selection of the most costeffective flood protection measures.

\section{ACKNOWLEDGMENT}

Part of this research was carried out on behalf of the Belgian Science Policy (BELSPO), in the framework of the program "Science for a Sustainable Development". The authors also gratefully acknowledge the Walloon Ministry of Facilities and Transport (MET) for the Digital Surface Model and other data.

\section{REFERENCES}

Boukhris, O., P. Baguis, P.Willems \& E.Roulin. 2007. Climate change impact on hydrological extremes along rivers and urban drainage systems. II.Study of climate change scenarios: K.U.Leuven - Hydraulics Section \& Royal Meteorological Institute of Belgium: 92

Chaudhry, M. H. 1993. Open-Channel Flow. Englewood Cliffs: Prentice Hall.

De Groof, A., W. Hecq, I. Coninx, K. Bachus, B. Dewals, M. Pirotton, M. El Kahloun, P. Meire, L. De Smet \& R. De Sutter. 2006. General study and evaluation of potential impacts of climate change in Belgium - Research report.

Dewals, B. 2006. Une approche unifiée pour la modélisation d'écoulements à surface libre, de leur effet érosif sur une structure et de leur interaction avec divers constituants: University of Liege. PhD thesis: 636.

Dewals, B. J., S. Detrembleur, P. Archambeau, S. Erpicum \& M. Pirotton. 2008a. Detailed 2D hydrodynamic simulations as an onset for evaluating socio-economic impacts of floods considering climate change (accepted). Proc. European Conference on Flood Risk Management - FloodRisk 2008. Rotterdam: Balkema.

Dewals, B. J., S. Erpicum, P. Archambeau, S. Detrembleur \& M. Pirotton. 2006. Depth-integrated flow modelling taking into account bottom curvature. J. Hydraul. Res. 44(6): 787-795.

Dewals, B. J., E. Giron, J. Ernst, W. Hecq \& M. Pirotton. 2008b. Integrated assessment of flood protection measures in the context of climate change: hydraulic modelling and economic approach. Environmental Economics. K. Aravossis, C. A. Brebbia \& N. Gomez: $10 \mathrm{p}$.

Dewals, B. J., S. A. Kantoush, S. Erpicum, M. Pirotton \& A. J. Schleiss. 2008c. Experimental and numerical analysis of flow instabilities in rectangular shallow basins. Environ. Fluid Mech. 8: 31-54.

Dushmanta, D., H. Srikantha \& M. Katumi. 2003. A mathematical model for flood loss estimation. J. Hydrol. 277: 24-49.

Ernst, J., B. J. Dewals, E. Giron, W. Hecq \& M. Pirotton. 2008. Integrating hydraulic and economic analysis for selecting flood protection measures in the context of climate change. Proc. 4th Int. Symp. on Flood Defence, Toronto, Canada: Institue for Catastrophic Loss Reduction.

Erpicum, S. 2006. Optimisation objective de paramètres en écoulements turbulents à surface libre sur maillage multibloc: University of Liege: 356.

Erpicum, S., P. Archambeau, S. Detrembleur, B. Dewals \& M. Pirotton. 2007. A 2D finite volume multiblock flow solver applied to flood extension forecasting. Numerical modelling of hydrodynamics for water ressources. P. García-Navarro \& E. Playán. Londres: Taylor \& Francis: 321-325.

Erpicum, S., B. J. Dewals \& M. Pirotton. 2008. Automatic shape optimization with GAs and a $2 D$ free surface flow solver. 4th Int. Conf. on Advanced Computational Methods in Engineering Liege, Belgium.

FloodSite. 2006. Guidelines for socio-economic flood damage evaluation: 170

Intergovernmental Panel on Climate Change. 2007. Climate change 2007: Synthesis Report - Summary for policymakers.

International Commission for the Protection of the Rhine. 2001. ICPR Rhine atlas.

Penning-Rowsell, E. C., C. Johnson, S. M. Tunstall, S. M. Tapsell, J. Morris, J. B. Chatterton, A. Coker \& C. Green. 2003. The Benefits of Flood and Coastal Defence: Techniques and Data for 2003: Flood Hazard Research Centre, Middlesex University.

Thieken, A. H., V. Ackermann, F. Elmer, H. Kreibich, B. Kuhlmann, U. Kunert, H. Maiwald, B. Merz, M. Müller, K. Piroth, J. Schwarz, R. Schwarze, I. Seifert \& J. Seifert. 2008. Methods for the évaluation of direct and indirect flood losses. Proc. 4th Int. Symp. on Flood Defence, Toronto, Canada: Institue for Catastrophic Loss Reduction.

Van der Sande, C. J., S. M. de Jong \& A. P. J. de Roo. 2003. A segmentation and classification approach of IKONOS2 imagery for land cover mapping to assist flood risk and flood damage assessment. International Journal of Applied Earth Observation and Geoinformation 4: 217-229. 